\title{
Surgical Management of Labyrinthine Fistula as a Complication of Cholesteatoma
}

Case

Report I'Otorhinolaryngology Unit, Department of Surgery, Faculty of Medicine and Health Sciences, University Putra Malaysia, 43400 Serdang, Selangor, Malaysia, ${ }^{2}$ OtorhinolaryngologyDepartment, Hospital Serdang, Jalan Puchong, 4300 Kajang, Selangor, Malaysia.

\begin{abstract}
Introduction: Labyrinthine fistula is an uncommon complication of chronic otitis media with cholesteatoma. Surgical management of labyrinthine fistula is challenging due to high risk of worsening labyrinthine function post-operatively. Case report: We report a case of a 25 -year old female presented with one-week history vertigo, vomiting and fever with left intermittent otorrhea for the past one year. High resolution computed tomography (HRCT) of the temporal bone showed total opacification of left mastoid cavity and dehiscence of left lateral semicircular canal (LSCC). Left Modified Radical Mastoidectomy (MRM) was performed and cholesteatoma was found within mastoid and middle ear cavities with dehiscence of arch of left LSCC wall with no perilymph leak observed.

Conclusion: The presence of vertigo with or without sensorineural hearing loss in patients with chronically discharging ear must raise suspicion of labyrinthine fistula. Definitive diagnosis can only be made intraoperatively. The current recommendation on management is open surgery with removal of cholesteatoma and sealing of fistula using soft tissue graft which provides favourable outcome.
\end{abstract}

Key Words: Cholesteatoma, chronic otitis media, labyrinthine fistula.

Received: 29 April 2020, Accepted: 08 February 2021

Corresponding Author: Zuraini Mohammad Nasir, Otorhinology Unit, Department of Surgery, Faculty of Medicine and Health Sciences, University Putra Malaysia, 43400 Serdang, Selangor, Malaysia, Tel.: +60198866448, E-mail: naninasir@gmail.com.

ISSN: 2090-0740, 2021

\section{INTRODUCTION}

Labyrinthine fistula is an uncommon complication of chronic otitis media with cholesteatoma. It results investibular and auditory complications such as vertigo and sensorineural hearing loss. The prevalence of labyrinthine fistula secondary to cholesteatoma has been consistent over the past decades with an incidence of $5-10 \%^{[1]}$. Surgical management of labyrinthine fistula is challenging due to high risk of developing worsening labyrinthine function and sensorineural hearing loss post-operatively. In this report, we discuss a case of chronic otitis media with cholesteatoma complicated with labyrinthine fistula. We further discuss mode of presentation, specific features on radiological investigations and current surgical management.

\section{CASE STUDY}

A 25-year old female with no underlying illness presented with one-weekhistory of vertigo, vomiting and fever with left intermittent otorrhea for the past one year. Patient appeared lethargicand spontaneous left-beating nystagmus was observed. Fistula test was positive and there was tenderness over left mastoid region. Facial nerve was intact. Left otoscopy examinationrevealed posterior external auditory canal (EAC)wall sagging with copious pus and granulation tissue obstructing tympanic membrane view. Right otoscopy examination was normal. Tuning fork test indicated left conductive hearing loss. Pure Tone Audiometry (PTA) showed left severe mixed hearing loss (Figure 1). 


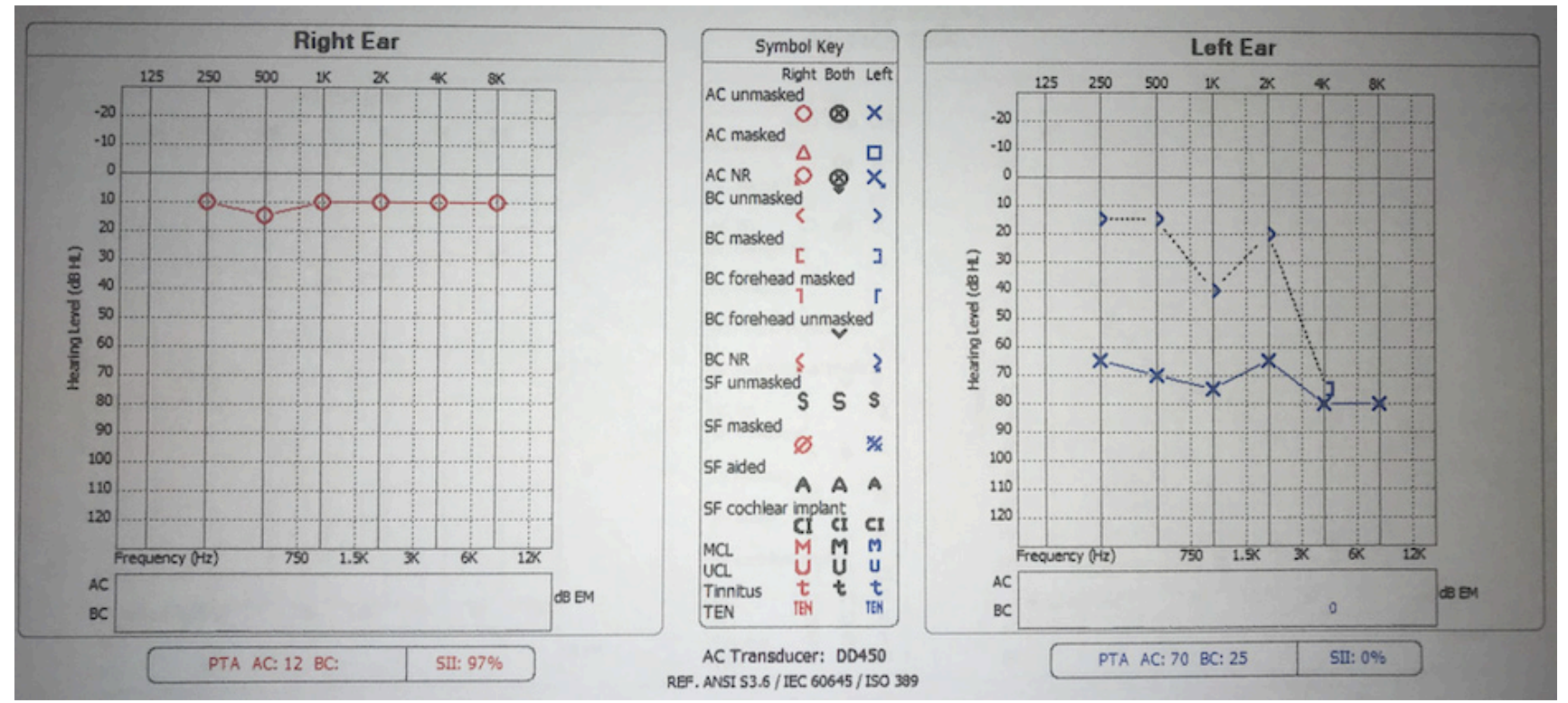

Fig. 1: Pure Tone Audiometry (PTA) showing left severe mixed hearing loss.

High resolution computed tomography (HRCT) of temporal bone showed total opacification of left mastoid and middle ear cavities, ossicular destruction and scutum erosion. There was focal wall dehiscence of the left lateral semicircular canal (LSCC) (Figure 2). The diagnosis of left chronic active otitis media with cholesteatoma complicated with labyrinthine fistula was made and patient was counselled for left Modified Radical Mastoidectomy (MRM). Potential risk of worsening hearing leading to 'dead ear' was explained. Intraoperatively,cholesteatoma sac was found within mastoid and middle ear cavities with erosion of posterior wall of EAC (Figure 3).

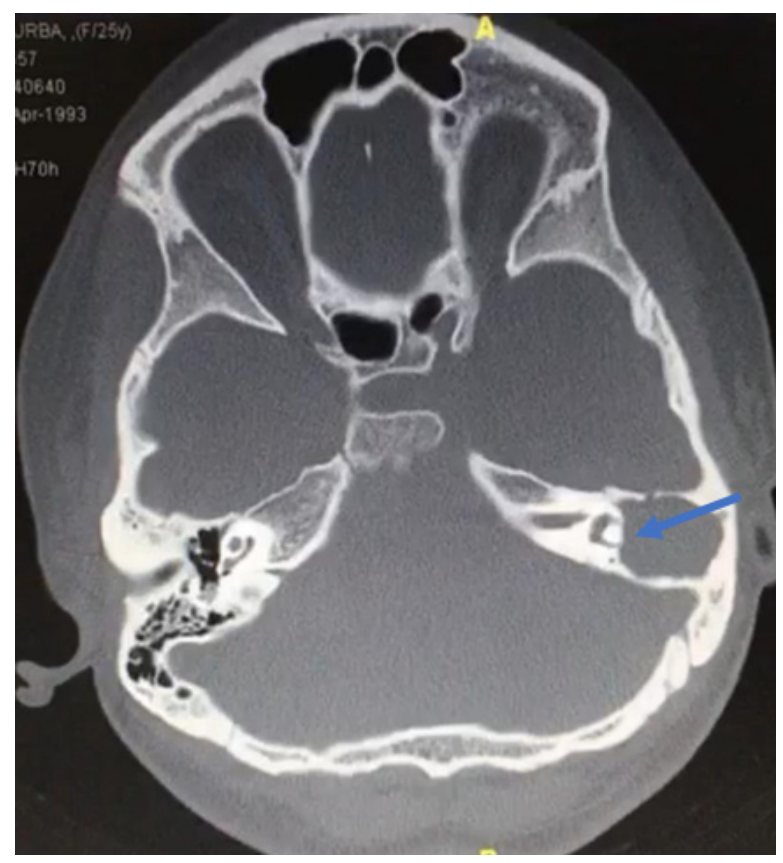

Fig. 2: Focal wall dehiscence of left lateral semicircular canal on high resolution computed tomography (HRCT) of temporal bone.

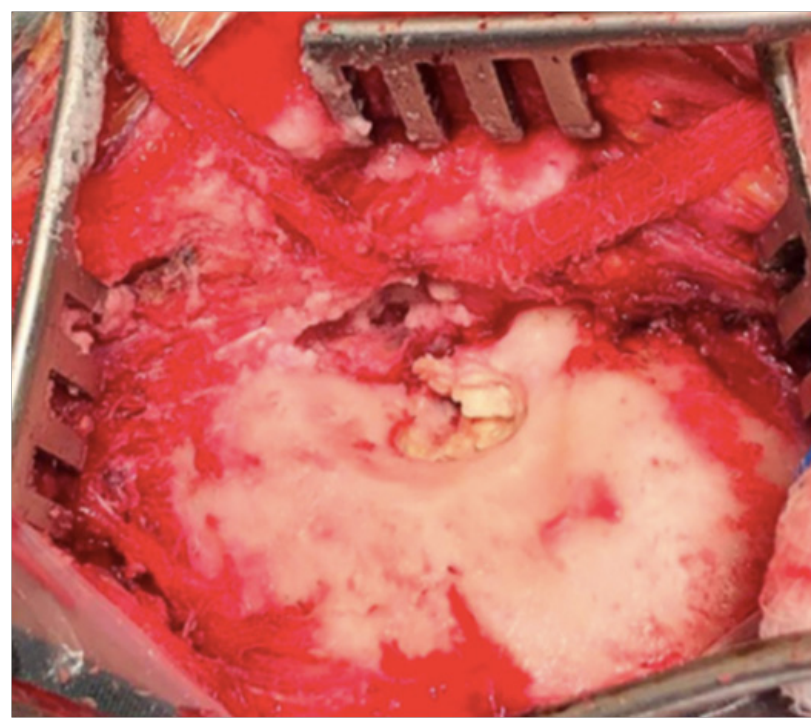

Fig. 3: Cholesteatoma sac within mastoid and middle ear cavities with erosion of posterior wall of EAC.

Arch of LSCC wall was dehiscent with no perilymph leak observed (Figure 4). Ossicles were absent and there was total tympanic membrane perforation. Cholesteatoma removed completely and temporalis fascia was used to cover the defect over LSCC. Tympanoplasty type IV was done using temporalis fascia graft. Post-operative period was uneventful and patient was discharged three days after operation. She was well at two weeks post-operative outpatient review with complete resolution of vertigo. At six-month outpatient review, there was no ear discharge or vertigo, no evidence of recurrent cholesteatoma and PTAshowed left profound mixed hearing loss (Figure 5). 


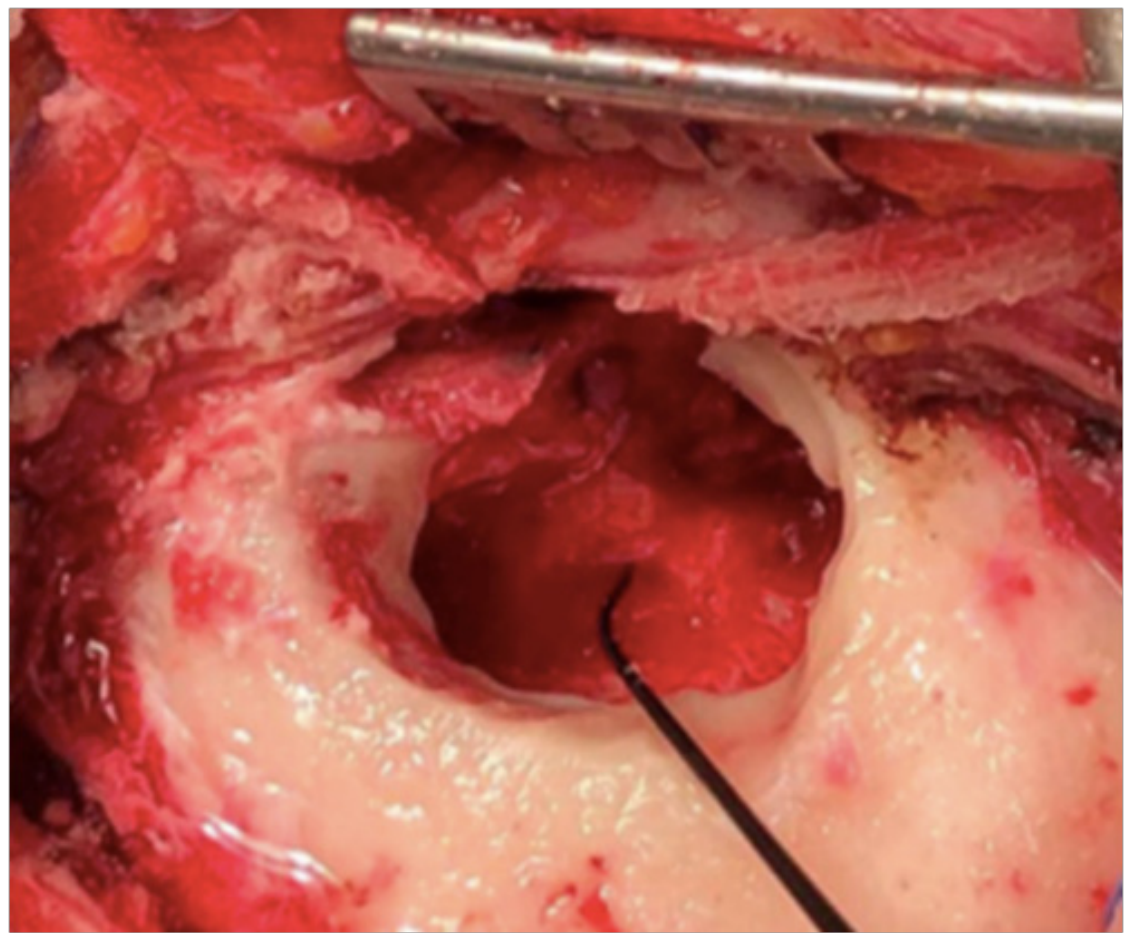

Fig. 4: Arch of left lateral semicircular canal was dehiscent with no perilymph leak observed.

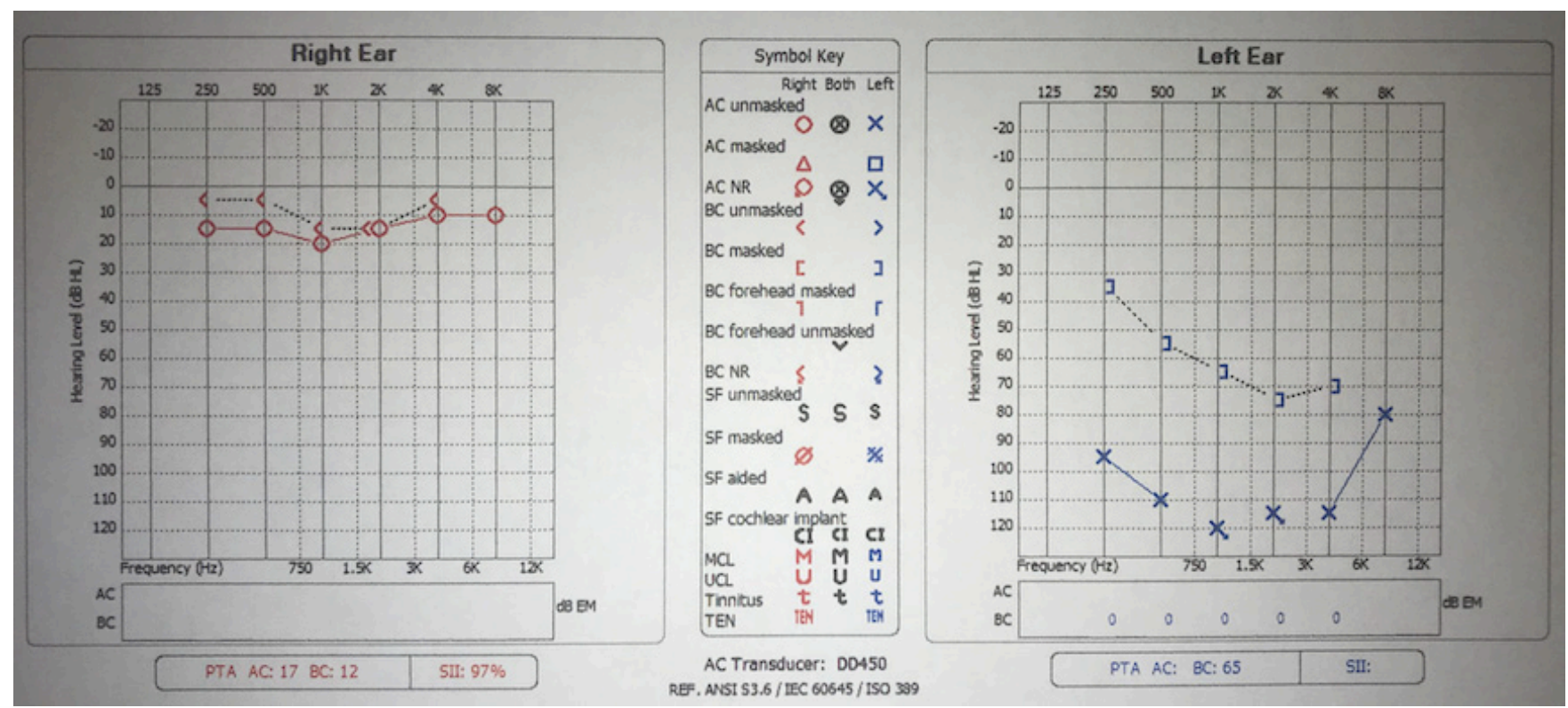

Fig. 5: Post-operativepure tone audiogram (PTA) at six months showed left profound mixed hearing loss.

\section{DISCUSSION}

Labyrinthine fistula occurs as a result of erosive loss of the overlying protective endochondral bone of labyrinth due to the production of collagenase by cholesteatoma ${ }^{[1,4]}$. In addition to that, mechanical pressure from cholesteatoma enhances granulation tissue formation, inducing osteoclast bone resorption ${ }^{[1]}$. This in turn allows mass-induced motion of the underlying endosteum, perilymphatic and endolymphatic compartments causing vestibular and sometimes auditory symptoms ${ }^{[4]}$. The most common site of fistula is lateral semi-circular canal (LSCC) in $87 \%$ of cases, promontory in $8 \%$, superior semi-circular canal (SSCC) in $6 \%$ and posterior semi-circular canal (PSCC) in $2 \%$ of cases ${ }^{[1]}$.

The most common symptom of labyrinthine fistula 
is short-lived episodic vertigo which is present in $64 \%$ of cases $(2,3)$. In addition to vertigo, patient can also present with sensorineural hearing loss. However, some patients may be asymptomatic due to the shielding effect of the membranous labyrinth and inner ear fluid by the cholesteatoma itself. Albeit being a diagnostic predictor, fistula test has been found to be positive in only $50 \%$ of cases $(2,3)$. False-negative fistula test can also occur when there is mass effect in the middle ear that prevents pressure transmission to inner ear or if the fistula is covered by a collection of epithelial debris ${ }^{[1,3]}$.

HRCT temporal bone is the gold standard investigation perform to assess the presence of labyrinthine fistula. It can also be used to assess extension of disease, identify other complications and plan for surgery. It has good sensitivity of detecting labyrinthine wall defect of $2.0 \mathrm{~mm}$ or more and is readily available. However, HRCT does not show the extension of fistula into endosteum which may affect surgical approach and functional prognosis. Apart from that, fistula may be missed on HRCT if large cholesteatoma is present ${ }^{[1]}$. Magnetic Resonance Imaging (MRI) of the temporal bone is the best modality to assess labyrinthine fistula. The suggestive features are reduction of space between cholesteatoma and membranous labyrinth, labyrinthine inflammation and partitioning of the perilymphatic fluid ${ }^{[6]}$. However, MRI has the disadvantage of not as readily available as HRCT. Despite radiological advances, definitive diagnosis of labyrinthine fistula can only be made during surgical exploration ${ }^{[3]}$.

Several classifications of labyrinthine fistula have been described in the literature. Dornhoffer and Milewski have introduced a classification which is based on the degree of involvement of labyrinth. A type I fistula is an erosion of the bony labyrinth with an intact endosteum. Type IIa is an opened perilymphatic space with undisturbed perilymph while type IIb has a disturbed perilymph. A Type III fistula is an opened perilymphatic space with destruction of the underlying membranous labyrinth ${ }^{[5]}$.

The aim of surgery is to eradicatedisease whilst preserving vestibular and cochlearfunctions. The choice of surgical treatment is controversial. Some advocatepreservation of overlying cholesteatoma over fistula and others advocatecomplete removal of cholesteatoma $^{[3]}$. Advocates for leaving matrix behind concluded that preservation of the matrix over labyrinthine fistula protects labyrinthine and cochlear function. The incidence of post-operative sensorineural hearing loss associated with removal of matrix has been cited to be between $6 \%$ and $37 \%{ }^{[5]}$. Furthermore, previous studies have reported spontaneous dissolution of cholesteatoma matrix with bony closure of the fistula and decreased size of the fistula in an uninfected state at second stage surgery ${ }^{[1]}$. Other authors argued that complete removal of cholesteatoma matrix should be attempted whenever possible as residual cholesteatoma may lead to further labyrinthine destruction. Palva et al. reported that one out of thirteen patients with residual cholesteatoma developed suppurative labyrinthitis and deafness ${ }^{[7]}$. Recentlythere has been a shift towards complete removal of cholesteatoma as the post-operative outcome has been found to be favorable in terms of eradication of diseaseand prevention of longterm complications of cholesteatoma ${ }^{[2]}$.

The consensus on surgical management of labyrinthine fistula is complete removal of cholesteatoma matrix from fistula site and grafting with soft tissue. This method is preferred because it provides safe and dry ear, prevents long term complication of cholesteatoma and avoids revision surgery ${ }^{[2]}$. The technique is first described by Dornhoffer in 1995. Once the fistula location is identified, the cholesteatoma is cut and remaining overlying matrix is left in place. After mastoid exenteration is completed, matrix is removed carefully ${ }^{[5]}$. Extreme caution is imperative during removal of cholesteatoma such as avoidance of direct suctioning of fistula and prevention accidental entry of blood or irritants into labyrinth ${ }^{[2]}$. The resulting bony defect is then quickly covered with bone dust and fibrin glue followed by soft tissue cover ${ }^{[5]}$. Good and adequate grafting of the fistula protects the labyrinth from caloric effect $^{[2]}$. Mastoid obliteration is also recommended to prevent recurrent cholesteatoma ${ }^{[1]}$.

Another controversy is whether canal wall down or two-staged canal wall up procedureshould be performed ${ }^{[5]}$. However, the surgical approach is more likely based on extension of cholesteatoma rather than the fistula $^{[6]}$. In selected cases with limited disease, canal wall up is optedwith second look surgery between 6-12 months post-operatively ${ }^{[2]}$.

Intraoperative use of corticosteroid hasalso beeninvestigated in the literature. The proposed mechanism of action is membrane stabilization propertyresulting inimprovedlabyrinthine and cochlear function. Intravenous corticosteroid must be administered upon opening the labyrinth to achieve maximum effect ${ }^{[5]}$. However, conflicting results have been reported. Dorhofferet. al reported that concomitant use of intravenous corticosteroid improves post-operative hearing in type $1 \mathrm{lb}$ and 111 fistulas ${ }^{[5]}$. Geerse et al. found no statistical difference in outcome ${ }^{[1]}$. Therefore, further prospective studies are required to determine its efficacy.

\section{CONCLUSION}

The presence of vertigo with or without sensorineural hearing loss in patients with chronically discharging ear must raise suspicion of labyrinthine fistula. Radiological investigations such as HRCT or MRI temporal bone may aid diagnosis. However, definitive diagnosis can only be made intraoperatively. The current recommendation on 
surgical management of labyrinthine fistula is complete removal of cholesteatoma matrix from fistula site and grafting with soft tissue.

\section{REFERENCES}

1. Geerse S, Wolf DMJF, Ebbens FA, Spronsen EV. Management of labyrinthine fistula: hearing preservation versus prevention of residual disease. Eur Arch Otorhinolaryngol. 2017;274(10):3605-3612.

2. Sagar P, Devaraja K, Kumar R, Bolu S, Sharma SC. Cholesteatoma Induced Labyrinthine Fistula: Is Aggressiveness in Removing Disease Justified? Indian J Otolaryngol Head Neck Surg. 2017;69(2):204-209.

3. Ludman H, Wright T. Diseases of the ear. 6th ed. Oxford University Press; 1998
4. Flint PW, Haughey BH, Lund VJ, Niparko JK, Richrdson MA, Robbins KT et al. Cummings Otolaryngology Head and Neck Surgery. 4th ed. Mosby Elsevier; 2005.

5. Dornhoffer JL, Milewski C. Management of the open labyrinth. Otolaryngol Head and Neck Surg. $1995 ; 112: 410-4$

6. Chafiki Z, Ait ELM, Rouadi S, Abada RL, Roubal M, Mahtar M. Management of the Labyrinthine Fistula in Chronic Otitis Media with Cholesteatoma. Global Journal of Medical Research: J Dentistry and Otolaryngology. 2016;16(1):23-25.

7. Palva T, Kärjä J, Palva A. Opening of the Labyrinth During Chronic Ear Surgery. Arch Otolaryngol. 1971;93(1):75-78 